This item was submitted to Loughborough's Research Repository by the author.

Items in Figshare are protected by copyright, with all rights reserved, unless otherwise indicated.

\title{
An analysis of thermal radiation in porous media under local thermal non- equilibrium
}

PLEASE CITE THE PUBLISHED VERSION

https://doi.org/10.1007/s11242-020-01408-x

\section{PUBLISHER}

Springer Verlag

VERSION

AM (Accepted Manuscript)

\section{PUBLISHER STATEMENT}

This is a post-peer-review, pre-copyedit version of an article published in Transport in Porous Media. The final authenticated version is available online at: https://doi.org/10.1007/s11242-020-01408-x.

\section{LICENCE}

CC BY-NC-ND 4.0

\section{REPOSITORY RECORD}

MoroFilho, Roberto C, and Weeratunge Malalasekera. 2020. "An Analysis of Thermal Radiation in Porous Media Under Local Thermal Non-equilibrium”. figshare. https://hdl.handle.net/2134/12037518.v1. 


\title{
An Analysis of Thermal Radiation in Porous Media under Local Thermal Non-equilibrium
}

\author{
R. C. MoroFilho ${ }^{1}$ \\ W. Malalasekera ${ }^{2}$ \\ ${ }^{1}$ Federal University of Technology, Paraná \\ 81280-340 - Curitiba - PR, Brazil \\ Corresponding author E-mail: robertoc@utfpr.edu.br \\ ${ }^{2}$ Wolfson School of Mechanical, Electrical and Manufacturing Engineering \\ Loughborough University \\ Loughborough LE11 3TU, United Kingdom
}

Keywords: Radiation, Porous Media, Local thermal non-equilibrium 


\begin{abstract}
The present work investigates the thermal radiation transport inside porous media under local thermal non-equlibrium conditions. Two different geometrical situations, a two-dimensional channel and a cylindrical geometry are considered in the analysis. Porous media application are generally associated with high-temperature combustion and radiative heat transfer is dominant. In this paper, by considering a stream of high-temperature flow in geometries filled with porous medium, the effect of thermal radiation on the solid and fluid phase temperature fields are calculated and analysed. Two radiative heat transfer techniques, the Discrete Ordinate Method (DOM) and the Finite Volume Method (FVM) are tested and the results are compared with a non-radiative model and with a model based Rosseland approximation. A sensitivity study for the variation of absorption and scattering coefficients and the effect on the solid and fluid phase temperature fields is also undertaken. The effect of the extinction coefficient on the Nusselt number is also examined. The temperatures obtained through the Rosseland model shows differences up to $+34 \%$ and $-26 \%$ compared to temperatures calculated with the DOM or FVM radiation calculation methods. The temperature fields obtained through DOM and FVM are very similar and some significant differences can only be seen in the cases where a very low number of angular directions are used.
\end{abstract}




\section{Nomenclature}

$c_{F}$

$c_{p}$

$d_{p}$

$\mathrm{d}_{\mathrm{m}}$

$D_{\text {diff }}$

G

h

$h_{v}$

$\mathbf{I}$

K

$k_{f}$

$k_{s}$

$\mathbf{K}_{\text {disp }}$

$\mathbf{K}_{e f f}$

$K_{r}$

$\mathrm{Nu}$

$\mathrm{p}$

$\mathrm{T}$

$\mathbf{u}_{D}$
Forchheimer coefficient

Constant pressure specific heat of mixture, $[\mathrm{kJ} / \mathrm{kg}-\mathrm{K}]$

Particle diameter $[\mathrm{m}]$

Mean pore diameter $[\mathrm{m}]$

Macroscopic diffusion tensor

Incident radiation function

Interfacial convective heat transfer coefficient, $\left[\mathrm{W} / \mathrm{m}^{2} \cdot \mathrm{K}\right]$

Volumetric heat transfer coefficient, $\left[\mathrm{W} / \mathrm{m}^{3} \cdot \mathrm{K}\right]$

Unit tensor

Permeability, $\left[\mathrm{m}^{2}\right]$

Fluid thermal conductivity, $[\mathrm{W} / \mathrm{m}-\mathrm{K}]$

Solid thermal conductivity, [W/m-K]

Dispersion conductivity tensor

Effective thermal conductivity tensor

Local Rosseland mean attenuation coefficient, $\left[\mathrm{m}^{-1}\right]$

Local Nusselt number

Pressure, $\left[\mathrm{N} / \mathrm{m}^{2}\right]$

Temperature, $[\mathrm{K}]$

Average surface velocity, $[\mathrm{m} / \mathrm{s}]$

Greek Characters

$\alpha_{\text {eff }} \quad$ Effective absorptivity 


$\begin{array}{ll}\varepsilon_{\text {eff }} & \text { effective emissivity } \\ \kappa & \text { absorption coefficient }\left[\mathrm{m}^{-1}\right] \\ \rho & \text { Dynamic viscosity, }[\mathrm{kg} /(\mathrm{m} . \mathrm{s})] \\ \sigma & \text { Density, }[\mathrm{kg} / \mathrm{m} 3] \text { or reflectivity, }[-] \\ \sigma_{s} & \text { Stefan-Boltzmann constant, }\left[\mathrm{W} /\left(\mathrm{m}^{2} \cdot \mathrm{K}^{4}\right)\right] \\ \phi & \text { scattering coefficient }\left[\mathrm{m}^{-1}\right] \\ \text { Subscripts } & \phi=\Delta V_{f} / \Delta V, \text { porosity } \\ \text { b } & \text { black body } \\ \mathrm{f} & \text { fluid } \\ \text { in } & \text { inlet } \\ \text { s } & \text { solid } \\ \text { W } & \text { wall }\end{array}$

\section{Introduction}

Practical situations that involve forced convection heat transfer in porous media are present in a wide range of engineering applications. These include packed beds for solardriven thermochemical processes, drying equipment, geothermal systems, catalyst layers in fuel cells, catalytic combustion systems, dehumidification equipment, energy storage units, porous media preheaters, nuclear reactors, equipment in petroleum industry, heat transfer enhancement devices and refrigeration systems are to name a few. In such applications, at high temperatures, the thermal radiative process become the dominant mode of heat transfer, as it occurs in radiant porous burners (Moro Filho and Pimenta 2011). Thus, the investigation and understanding of the thermal radiation process and its effects in porous 
media is crucial for the design and operation of porous media high-temperature applications.

Studies on macroscopic transport modeling of incompressible flows in porous media have been based on the volume-averaged methodology (continuum models) for either heat or mass transfer (Kaviany 1995; Nield and Bejan 2013). The accuracy of continuum models relies on the effective transport properties adopted in the calculations. The determination of the effective transport parameters is a very challenging task. The analysis of transport phenomena in reticulate porous ceramic (RPC), for example, is complex, some of the pores are open, some are closed and some are partially open or closed. Even samples from the same manufacturer show differences in the mean pore diameter. The geometry, shape and size distribution of the pores varies and depends on the method of manufacturing. Due to the complex morphology of the porous materials, the determination of the effective properties is a difficult task. However, some properties can be determined experimentally combined with advanced numerical techniques.

Recently, a combined experimental-numerical technique has been presented in the literature to determine effective properties. Petrasch et al. (2007) have used 3D digital representation of a reticulate porous ceramic (RPC) sample, generated by computer tomography $(\mathrm{CT})$ to determine radiative properties of the porous media. In their work computer tomography has been combined with Monte Carlo (MC) simulations to determine the total porosity, the surface-to-volume ratio, and the size of representative elementary volume (REV) for continuum. Other important properties such as extinction coefficient (beta), probability distribution functions and incident cosine have been determined through MC calculations. All radiative properties were determined assuming isotropy. The isotropic assumption has been justified by demonstrating that the extinction coefficient is directionally independent. 
There are other studies which have used experimental and theoretical approaches to calculate porous media properties. For example, Niya and Selvadurai (2017) have proposed a new approach for estimating the permeability of a planar porous structures. The approach estimates the pressure drop in various passages of the structure and then calculates the overall pressure drop based on the flow rate distribution. The predictions obtained using this approach were compared with the measured permeability values reported in the literature and the results obtained from other computational approaches. It was shown that the approach can predict the permeability values faster than CFD approaches with comparable accuracy. Other studies which have considered experimental and analytical methods for the determination of porous media properties include Frederking et al. (1986), Miguel and Serrenho (2007) and Ozgumus et al. (2014).

There are a number of experimental investigations which have considered convection heat transfer in porous media. These include the studies of Hwang and Chao (1994) and Jiang et al. (2004). Other studies which have considered modelling include the work of Yang and Vafai (2011), Ouyang et al. (2013) and Imani et al. (2012). In the analysis of porous media heat transfer, local thermal equilibrium (LTE) and local thermal nonequilibrium (LTNE) models can be used. The LTE model is valid when the heat exchange between the solid and fluid phases is high so that the local temperature difference between the two phases is negligible. When there is substantial heat transfer exchange between the fluid and solid phases within the porous medium the LTNE model needs to be used. Many practical applications required the LTNE model. For example, analysis of thermal radiation in a solar air receiver involving porous media has been considered by Wang et al. (2014) using LTNE representation.

The present paper utilizes the LTNE model for the analysis of radiative heat transfer in porous media in representative combustor conditions. Calculation involving combustion 
and radiative heat transfer in porous media is sensitive to porous media properties. A sensitivity analyses of the Rosseland mean attenuation coefficient has been presented by Moro Filho and Pimenta (2011) in problems involving porous burners. The authors considered the radiation in an optically dense medium and modelled it using the diffusion approximation, or the Rosseland model (Howell et al. 2011). The work showed that the optical properties of the materials have a strong influence on the pollutant emissions in porous burners. The analysis showed that, increasing the Rosseland mean attenuation coefficient $\left(K_{r}\right)$ by $50 \%$, increased the $\mathrm{CO}$ emissions at the exit of the reactor up to $34 \%$ and decreasing the $K_{r}$ by $50 \%$, decreased the $\mathrm{CO}$ emissions at the exit of the reactor up to $25 \%$. It is important to note that the problems considered were solved considering local thermal equilibrium between the solid and fluid phases and the diffusion approximation was used to model the radiative heat transport, which are considerable simplifications.

There are very few studies which have attempted to analyze thermal radiation heat transfer in porous media under local thermal non-equilibrium conditions coupled with conduction and advection. The work of Malico et al. (2000) studied radiative heat transfer under local thermal non-equilibrium condition in a problem involving combustion in porous media. They considered a porous medium as emitting, absorbing and isotropically scattering radiation and the discrete ordinate method was applied to solve the radiative transfer equation (RTE). In this work a number of different phase functions were tested and only the modified Henyey-Greenstein phase function showed significant differences when compared to isotropic scattering case. They concluded that perturbing the extinction coefficient and the scattering albedo greatly affects the temperature distribution. Comparing the results obtained considering radiation with a non-radiative model, they proved that radiation should not be neglected in porous burners. 
The work of Mahmoudi (2014) has presented an extensive analysis of a pipe filled with a porous material considering radiation in the modeling. In his study, Mahmoudi has considered the effects of, the porosity, solid and fluid thermal conductivity ratio, Darcy number and inertia parameter, on the fluid and solid phase on the non-dimensional temperature profiles. The work also presented an analysis of two different boundary conditions utilized for the solid and fluid energy equations. The work concluded that the non-radiative model leads to significant differences in results, up to $50 \%$, compared to the cases which considered thermal radiation.

The purpose of the present work is to investigate the radiative heat transport inside of a porous medium considering local thermal non-equilibrium condition. Forced convection heat transfer in tubes and channels filled with a porous material are considered as representative problems in the present analysis. The problems considered here are

representative examples for many practical porous media applications. The radiative transfer equation is solved through the Discrete Ordinate Method (DOM) and the Finite Volume Method (FVM) (Modest 2013; Howell et al. 2011). Further the results are compared with calculations using the Rosseland model and with a non-radiative model. A sensitivity study of absorption and scattering coefficients and the effect on the fluid and solid phase temperature fields are considered. The effect of the extinction coefficient on the Nusselt number is also examined.

\section{Macroscopic transport equations}

\subsection{Macroscopic continuity equations}

The macroscopic continuity equation governing flow in an incompressible fluid is given by:

$$
\nabla \cdot\left(\rho \mathbf{u}_{\mathrm{D}}\right)=0
$$


where, $\mathbf{u}_{\mathrm{D}}$ is the average surface velocity (also known as seepage, superficial, filter or Darcy velocity).

\subsection{Macroscopic momentum equation}

The heuristic macroscopic momentum equation utilized in this work is available in the literature (Kaviany 1995) and corresponds to an attempt by the scientific community to develop an equation, based on a volume-averaged treatment of the flow field, along the lines of Navier-Stokes equations. Another desirable characteristic of this heuristic equation is that it can describe both the momentum transport through the porous media as well as that in the clear flow region. The steady-state momentum equation is

$$
\nabla \cdot\left(\frac{\rho \mathbf{u}_{\mathrm{D}} \mathbf{u}_{\mathrm{D}}}{\phi}\right)=-\nabla(\phi p)+\mu \nabla^{2} \mathbf{u}_{\mathrm{D}}-\left[\frac{\mu \phi}{K} \mathbf{u}_{\mathrm{D}}+\frac{c_{F} \phi \rho\left|\mathbf{u}_{\mathrm{D}}\right| \mathbf{u}_{\mathrm{D}}}{\sqrt{K}}\right]
$$

The last two terms in equation (2), represent the Darcy-Forchheimer contribution. The Forchheimer coefficient, $c_{F}$, represents the deviation from Stokes flow and that in multidimensional flows, the extent of the inertial-core formation and flow separation. Ward (1964) recommends a value of $c_{F}=0.55$. It is presented as the Ergun coefficient by some authors (Kaviany 1995). The symbol $K$ is the porous medium permeability, $c_{F}$ is the form drag coefficient (Forchheimer coefficient), $p$ is the intrinsic (volume-averaged on fluid phase) pressure of the fluid, $\rho$ is the fluid density, $\mu$ represents the dynamic viscosity of the fluid and $\phi$ is the porosity of the porous medium. The following equations and expressions are used to obtained required properties.

\subsubsection{Permeability}

A study of the influence of the permeability on the pressure drop has been presented by Moro Filho (2014). He tested three different models to estimate the permeability and a value of the permeability determined by Petrasch et al. (2007) through direct pore level 
numerical simulation (DPLS). The value of permeability determined by DPLS is considered more accurate, but is related to a specific sample.

The correlation presented by Kuwahara et al. (1998), equation (3), was obtained numerically considering the flow within a spatially periodic array of circular rods. The permeabilities calculated with it result higher pressure drop than that calculated considering the permeabilities determined by Petrasch et al. (2007). The authors of the present work adopted it because, due to the simple geometry, it allows to calculate the permeability from the porosity and the particle diameter, what it is not possible in complex geometries. This makes the computational code more adjustable to different problems considering different porous media.

$$
K=\frac{\phi^{3}}{144(1-\phi)^{2}} d_{p}
$$

\subsection{Macroscopic Two-Energy Equations Model}

There are situations that the local thermal equilibrium hypothesis is not applicable. These situations are characterized through a considerable difference between the solid phase temperature and the fluid phase temperature generally occurring due to the low rate of heat transfer between the two phases or high heat generation in one phase. These situations require a two-energy equation model: one to the fluid phase and another to the solid phase.

The local thermal non-equilibrium assumption is considered in the present study. The macroscopic two-energy equation model (Malico et al. 2000; Kaviany 1995) is given by

$$
\begin{aligned}
& \left(\rho c_{p}\right)_{f} \nabla \cdot\left(\mathbf{u}_{D} T_{f}\right)=\nabla \cdot\left\{\mathbf{K}_{e f f, f} \cdot \nabla T_{f}\right\}+h_{v}\left(T_{s}-T_{f}\right) \\
& \text { and } \\
& \nabla \cdot\left\{\mathbf{K}_{e f f, s} \cdot \nabla T_{s}\right\}-h_{v}\left(T_{s}-T_{f}\right)=\nabla \cdot \mathbf{q}_{\mathbf{r}}
\end{aligned}
$$


represent the energy equation for the fluid and solid phase, respectively, where, $T_{f}$ and $T_{s}$ are the intrinsic volume average of the temperatures of the fluid phase and solid phase, $h_{v}$ is the volumetric heat transfer coefficient and $\mathbf{K}_{\text {eff,f }}$ and $\mathbf{K}_{\text {eff,s }}$ are the effective conductivity tensors for the fluid and the solid phase, respectively, given by:

$$
\begin{aligned}
& \mathbf{K}_{e f f, f}=\phi k_{f} \mathbf{I} \\
& \mathbf{K}_{e f f, s}=(1-\phi) k_{s} \mathbf{I}
\end{aligned}
$$

where, $k_{f}$ and $k_{s}$ are the thermal conductivities for the fluid and for the solid, respectively and $\mathbf{I}$ is the unit tensor. In this work, the effects of dispersion and tortuosity are neglected.

The energy equations of the solid and fluid phases are coupled through the convection term. There are many empirical correlations that can be used to calculate $h_{v}$. These correlations involve the Nusselt number and the Reynolds number. The choice of a characteristic length for correlating heat transfer data in terms of Nusselt and Reynolds numbers represent an important technical issue. Lengths based on average particle diameter $\left(d_{p}\right)$, mean pore diameter $\left(d_{m}\right)$, the square root of permeability $\left(K^{1 / 2}\right)$, the hydraulic diameter $\left(d_{h}\right)$ and others are found in the correlations presented in the literature (Kuwahara et al. 2001; Fu et al. 1998; Petrasch et al. 2007).

Fu et al. (1998) determined experimentally, through an inverse method, the volumetric heat transfer coefficient, $h_{v}$, of five different ceramics with different porosities at a wide range of Reynolds numbers. From their work, the authors developed an empirical correlation to obtain the volumetric Nusselt number as:

$$
N u_{v}=\frac{h_{v} d_{m}^{2}}{k_{f}}=C \operatorname{Re}^{m}
$$


Kuwahara et al. (2001) have presented a numerical procedure to determine the interfacial convective heat transfer coefficient, h, considering the flow through square rods placed in a staggered fashion. The square rods were maintained at a constant temperature. The Reynolds number was varied from $10^{-2}$ to $10^{3}$ and the porosity from 0.36 to 0.96 , whereas the Prandtl number was varied from $10^{-2}$ to $10^{2}$. From the numerical experiments, they provided the following expression:

$$
N u=\frac{h D}{k_{f}}=\left(1+\frac{4(1-\phi)}{\phi}\right)+\frac{1}{2}(1-\phi)^{1 / 2} \operatorname{Re}_{D}^{0.6} \operatorname{Pr}^{1 / 3}, \text { where, } 0.2<\phi<0.9
$$

The correlation presented by equation (9) was adopted in the present work. This correlation was created assuming a simple geometry which permits the analytical relation between the porosity and the permeability. Moreover, it was obtained for a large range of porosities and was validated comparing against the experimental data for packed beds assembled by Wakao and Kagei (1982).

Note that $\mathrm{Fu}$ et al. (1998) presented a Nusselt correlation considering the volumetric heat transfer coefficient, $h_{v}\left(\mathrm{~W} / \mathrm{m}^{3} \cdot \mathrm{K}\right)$. Kuwahara et al. (2001) and Wakao and Kagei (1982) presented Nusselt correlations considering interfacial convective heat transfer coefficient, $\mathrm{h}\left(\mathrm{W} / \mathrm{m}^{2} \cdot \mathrm{K}\right)$. The relation between $h_{v}$ and $\mathrm{h}$ is $h_{v}=a_{v} h$, where $a_{v}$ is the specific surface area (i.e., the area per unit volume).

\subsection{Thermal radiation modeling}

In the present study, the solid and fluid phases are treated as a single continuous homogeneous medium. The fluid is assumed to be transparent to radiation. The structure of the solid phase is considered to be gray. The radiation intensity of an absorbing, emitting and scattering grey medium along a path $\hat{s}$ is governed by the radiative transfer equation (Modest 2013; Howell et al. 2011): 


$$
\frac{d I(\vec{r}, \hat{s})}{d s}=-\beta(\vec{r}) I(\vec{r}, \hat{s})+S(\vec{r}, \hat{s})
$$

where the extinction coefficient and the source function are:

$$
\begin{aligned}
& \beta(\vec{r})=\kappa(\vec{r})+\sigma_{s}(\vec{r}) \\
& S(\vec{r}, \hat{s})=k(\vec{r}) I_{b}(\vec{r}, \hat{s})+\frac{\sigma_{s}(\vec{r})}{4 \pi} \int_{4 \pi} I\left(\vec{r}, \hat{s}^{\prime}\right) \Phi\left(\hat{s}^{\prime}, \hat{s}\right) d \Omega^{\prime}
\end{aligned}
$$

In equations (10), (11) and (12), $\vec{r}$ is the position vector, $\hat{s}$ is the unit vector describing the radiation direction and $I(\vec{r}, \hat{s})$ is the radiative intensity at a given location $\vec{r}$, in the direction $\hat{s}$.

After the radiative intensity field is calculated by solving the radiative transfer equation (RTE) for different directions, the divergent of the radiative heat flux $\left(\nabla \cdot \mathbf{q}_{\mathbf{r}}\right)$ is found by applying the equation (13) and is presumed to be constant in each sub-volume of the mesh. The equation (13) represents a radiative energy balance on an infinitesimal volume.

$$
\nabla \cdot \mathbf{q}_{\mathbf{r}}=\kappa\left(4 \pi I_{b}-\int_{4 \pi} I d \Omega\right)
$$

The second term on the right side of equation (13) represents the total intensity impinging on a point from all sides and is known as the incident radiation function $G$ (Modest 2013):

$$
G=\int_{4 \pi} I d \Omega
$$

In the case of an optically dense medium, radiation is attenuated travelling a short distance and for this situation, it is possible to transform the RTE into a diffusion relation (Howell et al. 2011). This is known as the Rosseland diffusion equation and is used in present work and compared with others methods in order to check the hypothesis of an optically dense medium. The term of the Rosseland model is included in the effective conductivity tensor for the solid phase as: 


$$
\mathbf{K}_{e f f, s}=(1-\phi)\left[k_{s}+\frac{16 \sigma\left(T_{s}\right)^{3}}{3 K_{r}}\right] \mathbf{I}
$$

\subsection{Boundary conditions}

Figure 1 presents the boundary conditions to the problem of a porous tube. The surfaces inlet and outlet of the packed bed radiates energy to the surrounding surfaces with a heat flux:

$$
q_{r}=-\left(\varepsilon_{e f f} \sigma T_{s}^{4}-\alpha_{e f f} \sigma T_{\infty}^{4}\right)
$$

where $\varepsilon_{e f f}$ is effective emissivity, $\alpha_{e f f}$ is the effective absorptivity of the porous bed and $T_{\infty}$ is the ambient temperature. The $\varepsilon_{e f f}$ and $\alpha_{e f f}$ are made equal to simplify the solution.

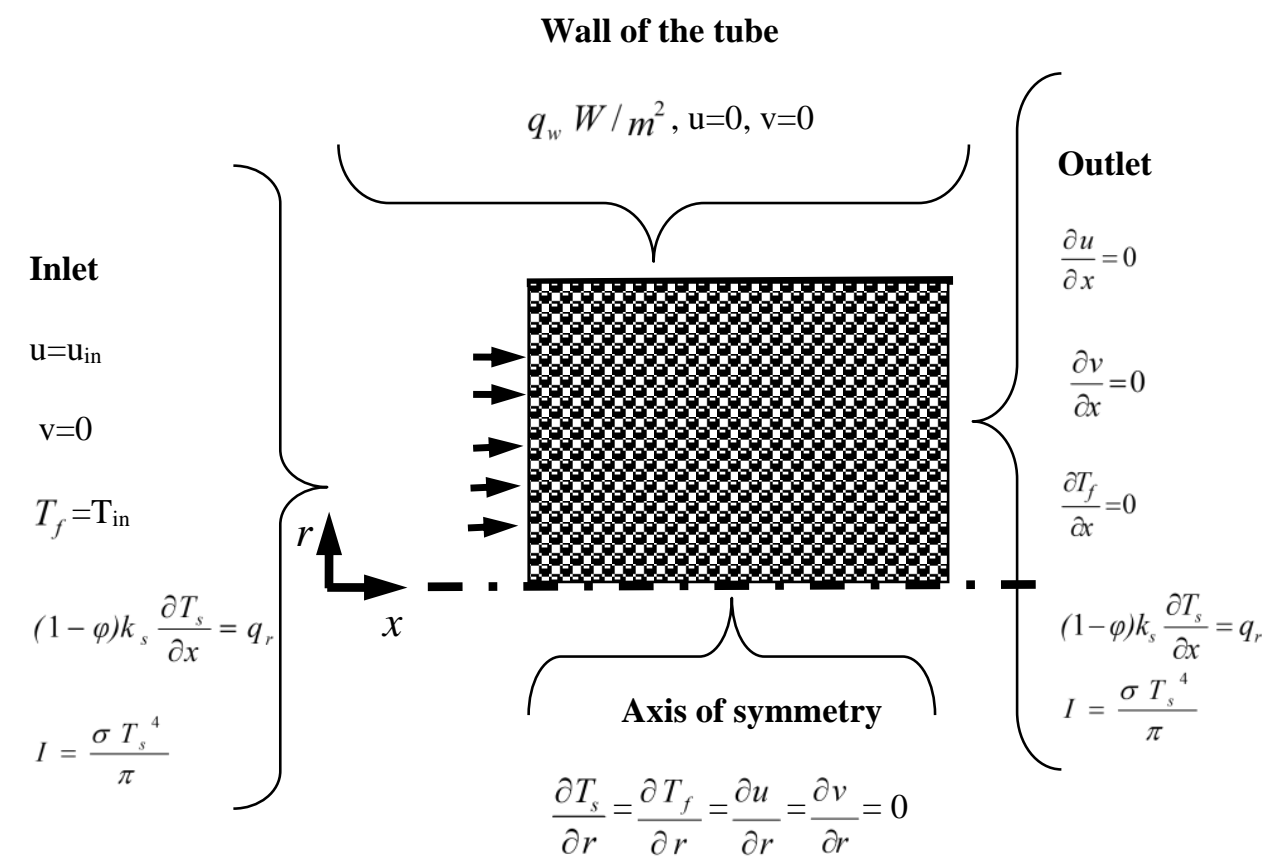

Figure 1. Boundary conditions for the tube

The fluid and solid boundary condition at the wall considers constant heat flux as follows:

$$
-\left.\phi k_{f} \frac{\partial T_{f}}{\partial r}\right|_{\text {wall }}=q_{w}
$$




$$
-\left.(1-\phi) k_{s} \frac{\partial T_{s}}{\partial r}\right|_{\text {wall }}=q_{w}
$$

Additionally, the wall of the tube is assumed to be opaque, diffusely emitting and reflecting, where the intensity $I$ is:

$$
I=\varepsilon_{w} I_{w}+\frac{\rho_{w}}{\pi} \int_{n \cdot s<0} I|n \cdot \mathrm{s}| d \Omega
$$

The wall heat transfer coefficient based on a pipe is defined by:

$$
h=\frac{q_{w}}{T_{f, \text { wall }}-T_{f, m}}
$$

Where $T_{f, \text { wall }}$ is the fluid temperature at the wall and the mean temperature $T_{f, m}$ is defined as:

$$
T_{f, m}=\frac{2}{U_{m} R^{2}} \int_{0}^{R} u T_{f} r d r
$$

The mean velocity $U_{m}$ is defined as:

$$
U_{m}=\frac{2}{R^{2}} \int_{0}^{R} u r d r
$$

The fluid Nusselt number based on the pipe radius and on the fluid effective conductivity is presented as:

$$
N u_{f}=\frac{h(2 R)}{\phi k_{f}}
$$

\section{The numerical model}

In the solution process, the governing equations are discretized using the finite volume method (Versteeg and Malalasekera 2007). The resulting system of algebraic equations are then solved through the semi-implicit procedure using the Stone algorithm (Stone 1968). The SIMPLE algorithm for the pressure-velocity coupling is used to correct both the pressure and the velocity fields (Patankar 1980). The process starts with the solution of the two momentum equations. Then the velocity field is adjusted in order to satisfy the 
continuity principle. This adjustment is obtained by solving the pressure correction equation. For all cases, a relative residual convergence criterion of $10^{-5}$ was used. The grid effects on the solutions were examined by increasing the number of nodes and verifying the solutions until the results no longer changed in a specified tolerance. The results presented here are with meshes that produced grid independent solutions.

The meshes utilized in the problems of the channel and of the tube are different. In the channel problem it was employed a mesh of $122 \times 34$, refined close the south and north boundaries. In the case of the tube, due to the high gradients in the field of the divergence of the radiative heat flux, it was necessary to use a finer mesh with $222 \times 34$ elements and refined close the wall and in the region of the exit.

In the present work, two different methods were employed to solve the RTE: Finite Volume Method (FVM) (Raithby and Chui 1990; Chai et al. 1994) and Discrete Ordinate Method (DOM) (Chandrasekhar 1960; Fiveland 1984; Truelove 1987). The DOM and the FVM formulations are similar for Cartesian coordinates despite the development is based on different concepts. As mentioned earlier the Rosseland diffusion equation is also applied to obtain solutions in order to check the hypotheses of the porous medium be considered optically thick.

\subsection{Discrete ordinate method}

In the discrete ordinate method (DOM), the RTE, equation (10), is solved for a set of $n$ different directions $\hat{s}_{i} \quad i=1,2,3 \ldots . . n$. Each direction is associated with a solid angle where the radiative intensity is assumed to be constant. The integrals over directions are replaced by a numerical quadrature. The method is an extension of the two-flux method developed by Schuster (1905) and Schwarzschild (1906). According to Chandrasekhar (1960), the division of the radiation field in just two streams is far too crude to include the effects of the anisotropic scattering and the division of the radiation field into more than just two 
streams would retain the basic idea, and preserve the fundamental relationships in all orders of approximation. The DOM method is further described by Modest (2013) and Howell et al. (2011).

In the DOM, equation (10) is solved for a set of $n$ different directions $\hat{s}_{i}$ and the integrals over direction are replaced by numerical quadratures. Hence the equation (10) is approximated by a set of $n$ equations,

$$
\hat{s}_{i} \cdot \nabla I\left(\vec{r}, \hat{s}_{i}\right)=-\beta I\left(\vec{r}, \hat{s}_{i}\right)+\kappa(\vec{r}) I_{b}(\vec{r})+\frac{\sigma_{s}(\vec{r})}{4 \pi} \sum_{j=1}^{n} w_{j} I\left(\vec{r}, \hat{s}_{j}\right) \Phi\left(\vec{r}, \hat{s}_{i}, \hat{s}_{j}\right), \quad i=1,2, \ldots, n
$$

where the $w_{j}$ are the quadrature weights associated with the directions $\hat{s}_{i}$.

\subsection{Finite volume method}

The finite volume method (FVM) was reported by Raithby and Chui (1990) and provides an angular discretization that allows to specify an angular grid according to the characteristics of the problem. The FVM applied in space and in the direction (solid angle) ensures the conservation of the radiative energy within a control angle, control volume and globally for any angular and spatial grid.

The integration of the equation (10) over the volume element $\Delta V$ and over the solid angle $\Delta \Omega^{k}$ leads to

$$
\int_{\Delta \Omega^{k}} \int_{\Delta A} I^{k}\left(\hat{s}^{k} \cdot \hat{n}\right) d A d \Omega^{k}=\int_{\Delta \Omega^{k}} \int_{\Delta V}\left(-\beta I^{k}+S^{k}\right) d V d \Omega^{k}
$$

Following the practice of the control volume approach, the radiative intensity over a control volume and solid angle remain constant, the integrals can be replaced by summations:

$$
\sum_{i=1}^{4} I_{i}^{k} \Delta A_{i} \int_{\Delta \Omega^{k}}\left(\hat{s}^{k} \cdot \hat{n}_{i}\right) d \Omega^{k}=\left(-\beta I^{k}+S^{k}\right) \Delta V \Delta \Omega^{k}
$$

where

$$
S^{k}=k I_{b}+\frac{\sigma_{s}}{4 \pi} \sum_{j=1}^{n} I^{j} \Phi^{j k} \Delta \Omega^{j}
$$


In equation (27) $\Phi^{j k}$ is the average scattering phase function from control angle $k$ to control angle $j$.

After some manipulation of equation (26) and applying the step discretization scheme (Modest 2013), leads to an algebraic equation relative to the radiative intensity in a node of the mesh for a specific direction $k$. There will be so many equations as the number of nodes and directions. Details of the FVM are described by Chai et al. (1994) and by Chui et al. (1992).

\section{Results and discussion}

In this work, two representative geometrical situations involving porous media heat transfer are considered. The first problem is a two-dimensional channel filled with a porous medium. The second problem considered is a cylindrical geometry filled with a porous medium. In both cases, the flow enters the geometry from outside at a fixed temperature and leaves from the other side. A constant heat flux is assumed at the walls of the channel. A sensitivity analysis is presented through three different cases: Case 1, Case 2 and Case 3. A constant heat flux at the wall is adopted in all simulations.

\subsection{The porous channel}

Figure 2 represents a channel with a porous material. The parameters and thermophysical properties adopted in the simulations are presented in Table 2 . The fluid medium is considered to be air. The length of the geometry, $L$, is $50 \mathrm{~cm}$ and the height $H$ is 10 $\mathrm{cm}$. 


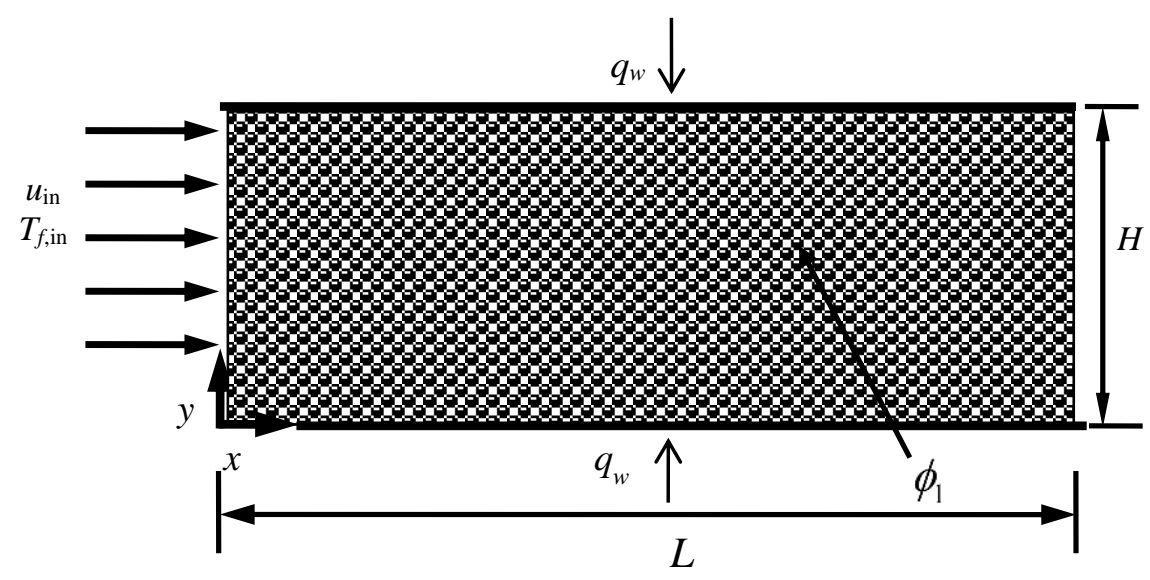

Figure 2. Geometry of a porous channel and the coordinate system

First, the problem is considered as a non-radiative case and solution is obtained with the geometry shown in Figure 2. Figure 3 presents the fluid and solid phase temperatures in the cross-section of the channel considering a fully developed flow for a non-radiative case. The solution is compared against the exact solution of Yang and Vafai (2010). Results are compared at the center of the domain. It can be seen that the numerical solution and the exact solution agree very well confirming that the solution methodology used in the present calculation is valid and accurate. The maximum difference between the temperatures of the exact solution and the numerical results is around $0.7 \mathrm{~K}$ hence the numerical solution accuracy is quite good. 


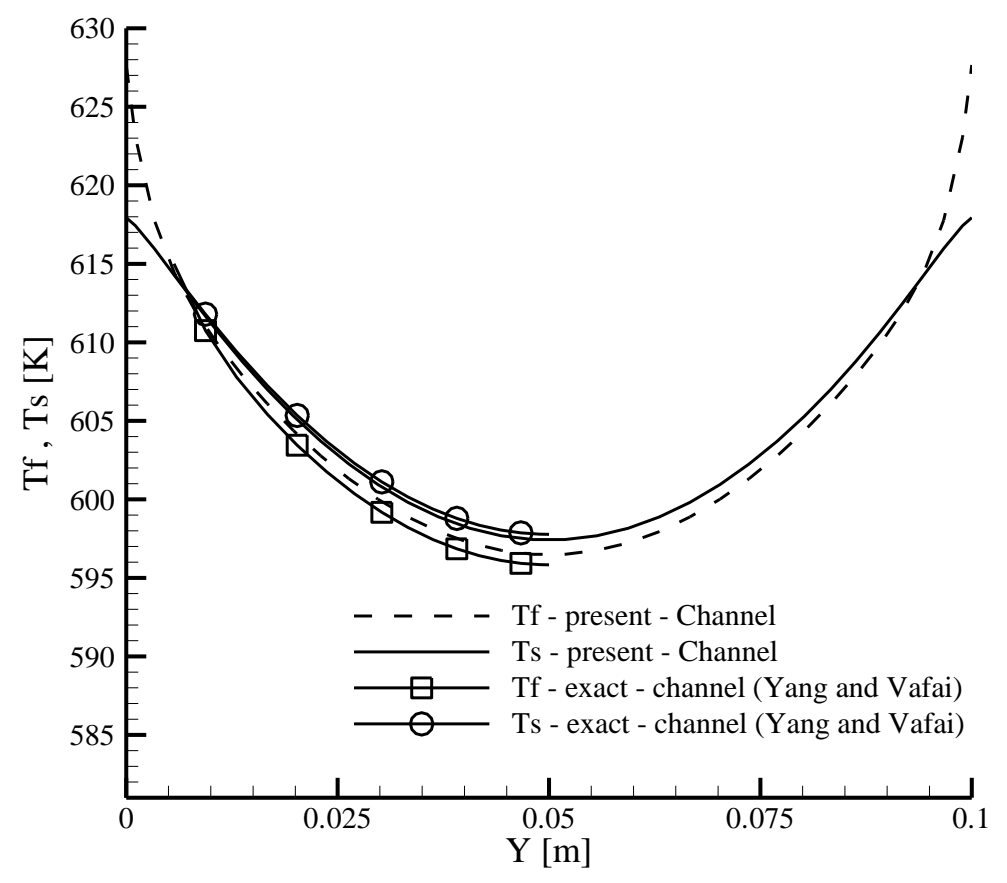

Figure 3. Comparison of the numerical results for the fluid and solid temperatures along the $y$-direction of the channel and the exact fluid and solid temperatures obtained by Yang and Vafai (2010) for a channel in the fully developed region, $q_{w}=100 \mathrm{~W} / \mathrm{m}^{2}$.

The next case, radiation in the solid phase is taken into consideration. The medium is considered to be absorbing, emitting and non-scattering. The numerically calculated temperature fields of the fluid and solid phases are presented in Figure 4. In this case, the spatial domain was divided into $122 \times 34$ control volumes with the mesh refined close to the walls. The RTE was solved through the finite volume method with the angular space subdivided into $14 \times 12$ control angles with uniform $\Delta \phi$ and $\Delta \theta$. The step scheme was used in all simulations. The absorption coefficient was specified as $\kappa=540 \mathrm{~m}^{-1}$. The field of the predicted incidence radiation is presented in Figure 5. The incidence radiation corresponds to the total intensity impinging on a point from all sides. 

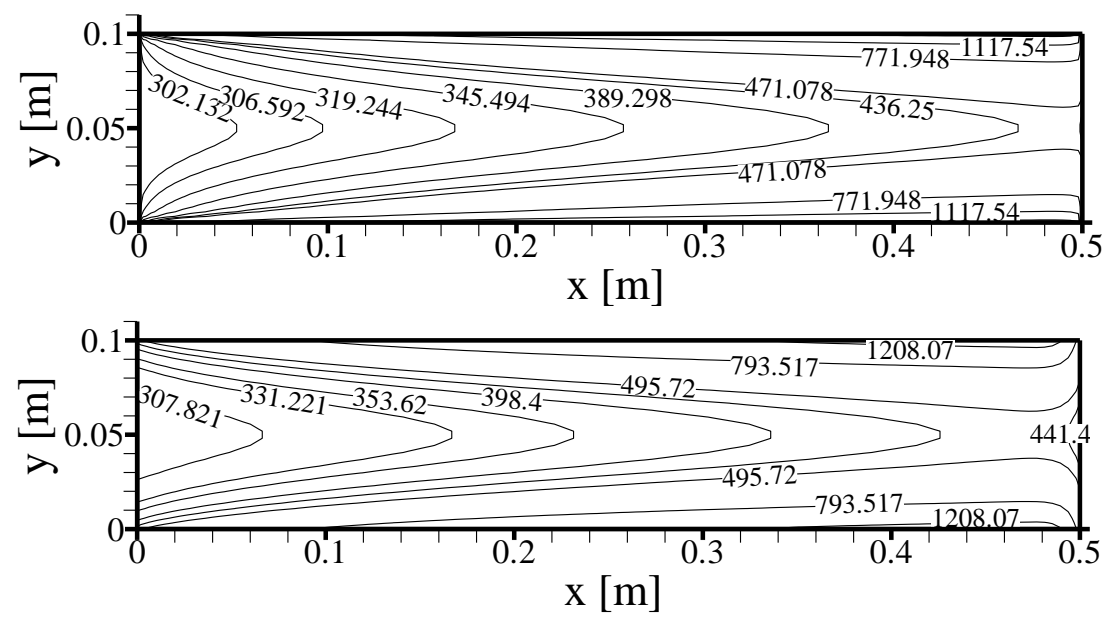

Figure 4. Fields of predicted temperature $(\mathrm{K})$ of the fluid phase (a) and solid phase (b) in a channel obtained through the method of FVM with an angular discretization $\mathrm{N}_{\phi}=14$ and $\mathrm{N}_{\theta}=12, \mathrm{q}_{\mathrm{w}}=5000 \mathrm{~W} / \mathrm{m}^{2}$.

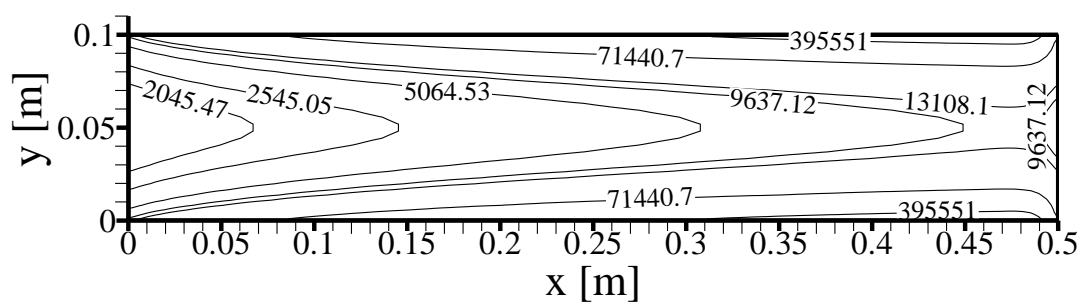

Figure 5. Field of predicted incidence radiation in a channel obtained through the method of FVM with an angular discretization $\mathrm{N}_{\phi}=14$ and $\mathrm{N}_{\theta}=12, \mathrm{q}_{\mathrm{w}}=5000 \mathrm{~W} / \mathrm{m}^{2}$.

The radiative heat transport is modelled including the divergent of the radiative heat flux as a source term in the solid energy equation. The fluid was considered transparent to radiation. In this case, it is important to observe the field of $\vec{\nabla} \cdot \vec{q}_{r}$ because high gradients exist in the field of $\vec{\nabla} \cdot \vec{q}_{r}$. In order to validate the calculation of the $\vec{\nabla} \cdot \vec{q}_{r}$, a number test cases were run. The problem consisting in a rectangular enclosure with cold, black walls and a purely absorbing medium maintained at an emissive power of unity was chosen since an exact solution exists presented by Shah (1979) and solved numerically by Fiveland (1984). It was tested changing the emissivity and comparing the results obtained with DOM and FVM with the exact and numerical results presented by Shah (1979) and Fiveland (1984), respectively. 
It can be seen in figure 7 the field of the divergence of the radiative heat flux in a channel. The analyses of this distribution help to study mesh refinement. In problems which the boundary conditions consider high heat flux on the walls, the temperature distributions are very high and the gradients of the divergence of the radiative heat flux are very high too. The high gradients demand meshes more refined.

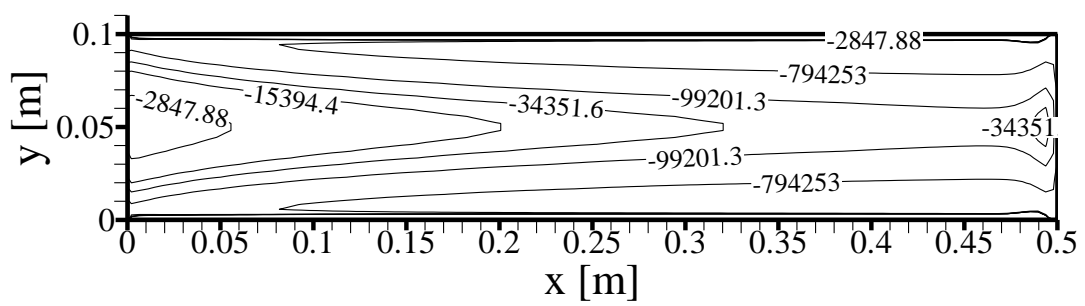

Figure 6. Field of predicted divergence of the radiative heat flux in a channel obtained through the method of FVM with an angular discretization $\mathrm{N}_{\phi}=14$ and $\mathrm{N}_{\theta}=12, \mathrm{q}_{\mathrm{w}}=5000 \mathrm{~W} / \mathrm{m}^{2}$.

Figure 7 shows a comparison of fluid and solid temperatures profiles obtained through the discrete ordinate method (DOM) and the finite volume method (FVM) considering a medium that is absorbing, emitting radiation, but non-scattering. The profiles were obtained in the center of the channel. The results with DOM and with the FVM are also compared with the results obtained with a non-radiative model and the Rosseland model which treat the medium to be optically dense. 


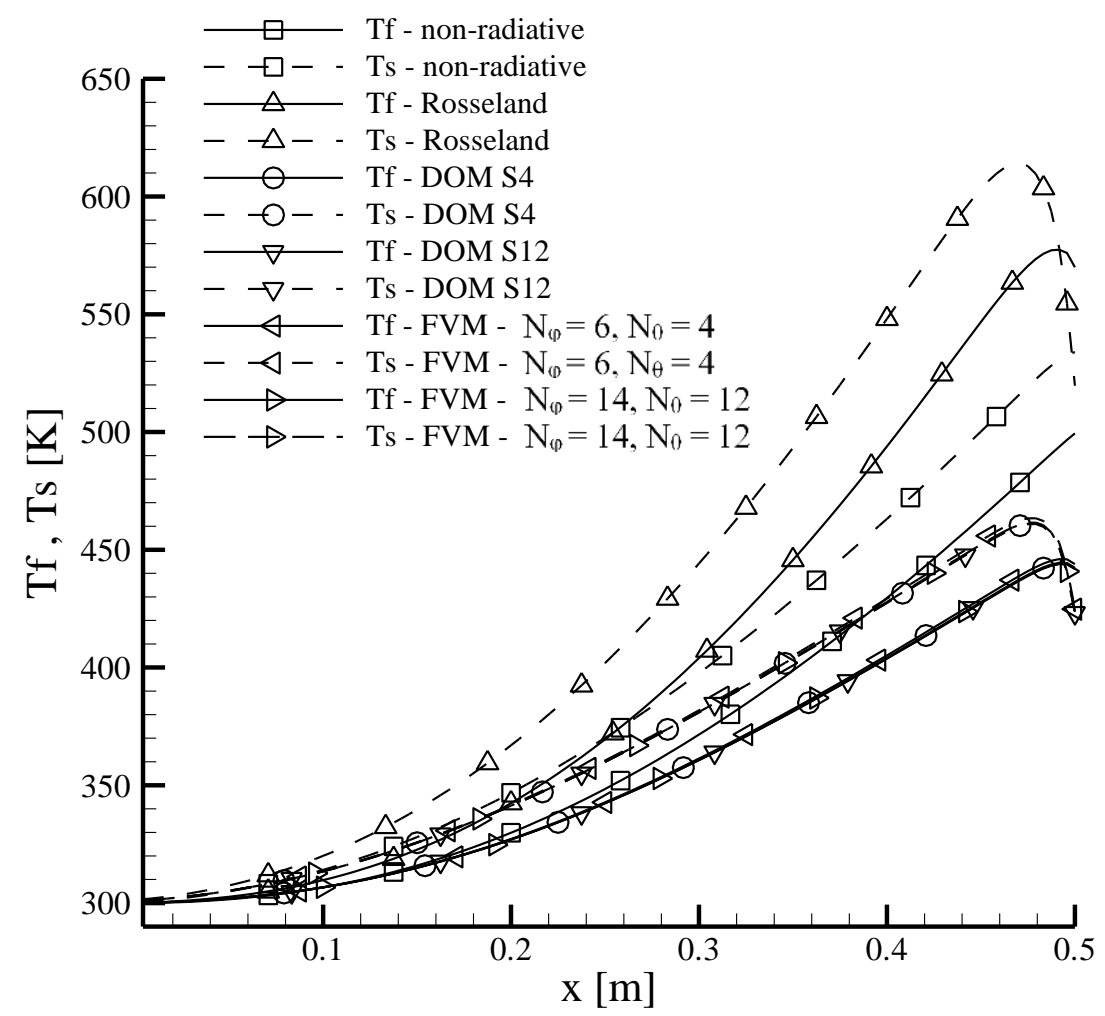

Figure 7. Temperature profiles in the center line of a porous channel obtained through the methods of FVM, DOM and Rosseland compared with the results of the non-radiative case, $\mathrm{q}_{\mathrm{w}}=5000 \mathrm{~W} / \mathrm{m}^{2}$.

Here DOM and FVM methods are tested with different angular discretization as well. The results obtained with the DOM and with the FVM are very close. The maximum difference in the temperature field, when comparing S12 with an angular discretization of $14 \times 12$, is $+0.26 \%$. However, the two methods show a larger difference in the cases where a very low number of angular directions are used. The maximum difference in the temperature field, when comparing S4 with an angular discretization of $6 \mathrm{x} 4$, is $+0.81 \%$.

In some regions of the channel, the temperatures of the Rosseland model are higher than the temperatures obtained with DOM or FVM and, in other regions, are lower. Therefore, there are positive and negative differences. The results obtained with Rosseland model differs up to $+34 \%$ and $-26 \%$ from the results obtained solving the R.T.E. through DOM or FVM. According to Howell et al. (2011) the diffusion approximation requires the intensity to be nearly isotropic within the medium, a situation that can satisfy this in 
interior of an optically thick medium, will not be valid near boundaries. Therefore the diffusion approximation loses accuracy near boundaries (Modest 2013). The hightemperature gradients near the walls cause the great differences between the results obtained with Rosseland and that obtained through FVM and DOM.

The temperatures of the two phases obtained through a non-radiative model shows high differences compared to the results with radiation. The maximum differences are $+147.2 \%$ and $-33.0 \%$. The differences between the results of DOM and FVM are small, therefore it was compared the DOM and FVM with the non-radiative model considering DOM or FVM as references. The maximum differences are $+147.2 \%$ and $-33.0 \%$, which means that there is a node of the mesh where the temperature calculated through the nonradiative model is $147.2 \%$ higher than that calculated with the FVM or DOM. It is compared with DOM and FVM and it is just presented the maximum differences, even because the results of FVM and DOM are very close. In the same way, there is a node in the mesh where the temperature calculated through the non-radiative model is $33.0 \%$ lower than that calculated with the FVM or DOM.

The temperature field obtained with the non-radiative model is just lower than that considering radiation in a small region close to the wall and near the inlet. In the case with radiation, there is a loss of energy by radiation at the inlet and the exit, the transport of heat through the channel is faster than the case without radiation, while the heat transfer through the wall is the same in both cases $\left(q_{w}\right)$. This leads to the temperatures fields in the radiative cases to be lower than that of the non-radiative case. The highest difference of $147 \%$ between the radiative and non-radiative model occurs in the region close to the wall at the exit. In this region, the temperature close to the wall is high due to considerable loss by radiation at the exit, which is not present in the case without radiation. 
To study the influence of the selected properties on the radiative field, a sensitivity analysis was performed considering a medium that absorbs, emits and scatters radiation. The absorption and scattering coefficient were perturbed by \pm 50 percent. The base values adopted for the optical properties were $\kappa=540 \mathrm{~m}^{-1}$ and $\sigma=2160 \mathrm{~m}^{-1}$. These are representative values for practical porous medium reported by Chaffin et al. (1991) and correspond to a zirconia foam.

The absorption coefficient of the porous medium, $\kappa$, was perturbed \pm 50 percent. The influence of the variations of $\kappa$ on the fluid and solid phase temperatures profiles are shown in Figure 8 . Increasing $\kappa$ by $50 \%$ causes an increase up to $1.24 \%$ on the solid phase temperature. Decreasing the $\kappa 50 \%$ causes a decrease up to $2.11 \%$ on the solid phase temperature.

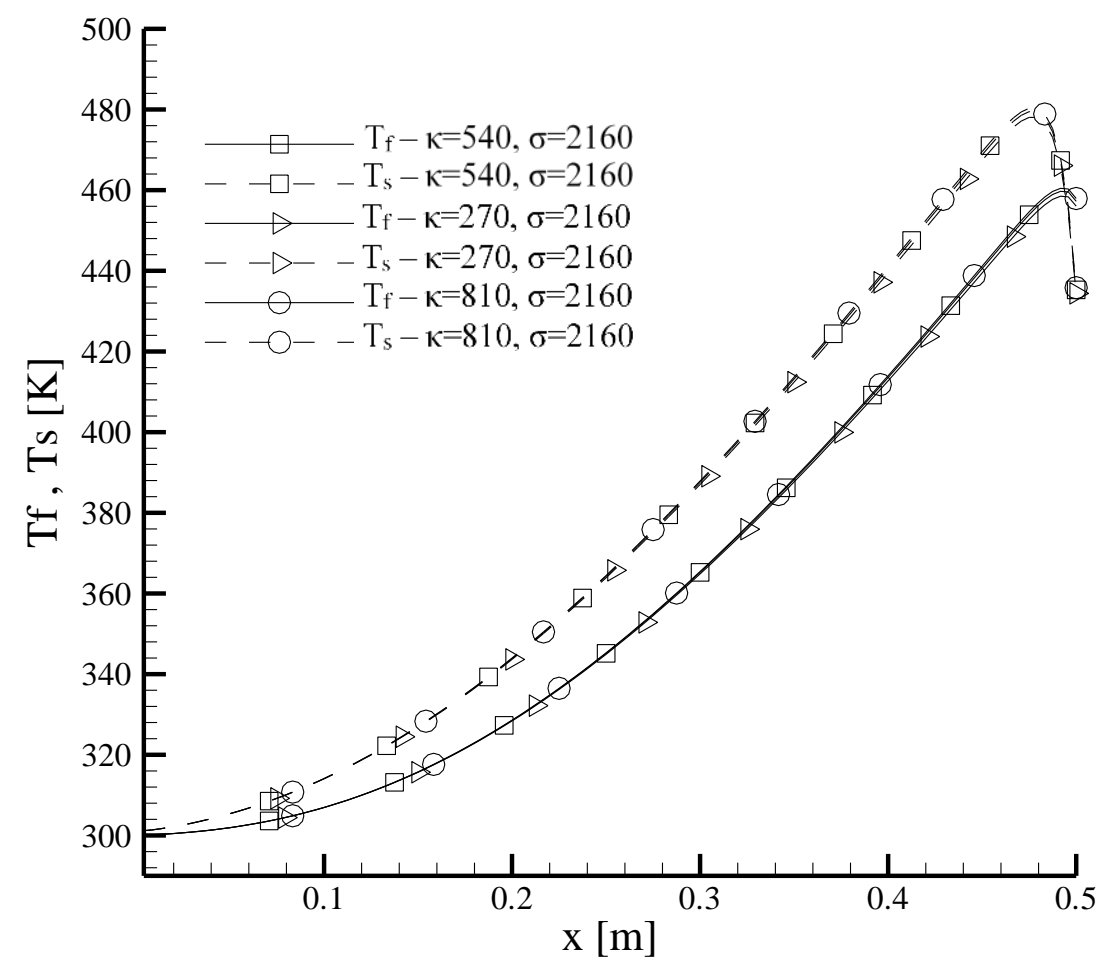

Figure 8. Temperature profiles of the fluid and solid phases in the center line of a porous channel obtained changing the absorption coefficient $\pm 50 \%$. It was utilized FVM with an angular discretization $\mathrm{N}_{\phi}=14$ and $\mathrm{N}_{\theta}=12$ considering isotropic scattering, $\mathrm{q}_{\mathrm{w}}=5000 \mathrm{~W} / \mathrm{m}^{2}$. 
The effect of the variations of the scattering coefficient $\sigma$ on the fluid and solid phase temperatures profiles are shown in the Figure 9. Increasing $\sigma$ by $50 \%$ causes an increase up to $0.54 \%$ of the solid phase temperature. Reducing $\sigma$ by $50 \%$ decreases up to $1.22 \%$ the solid phase temperature. The resulting maximum change in the temperature fields due to above changes in properties are shown in Table 1.

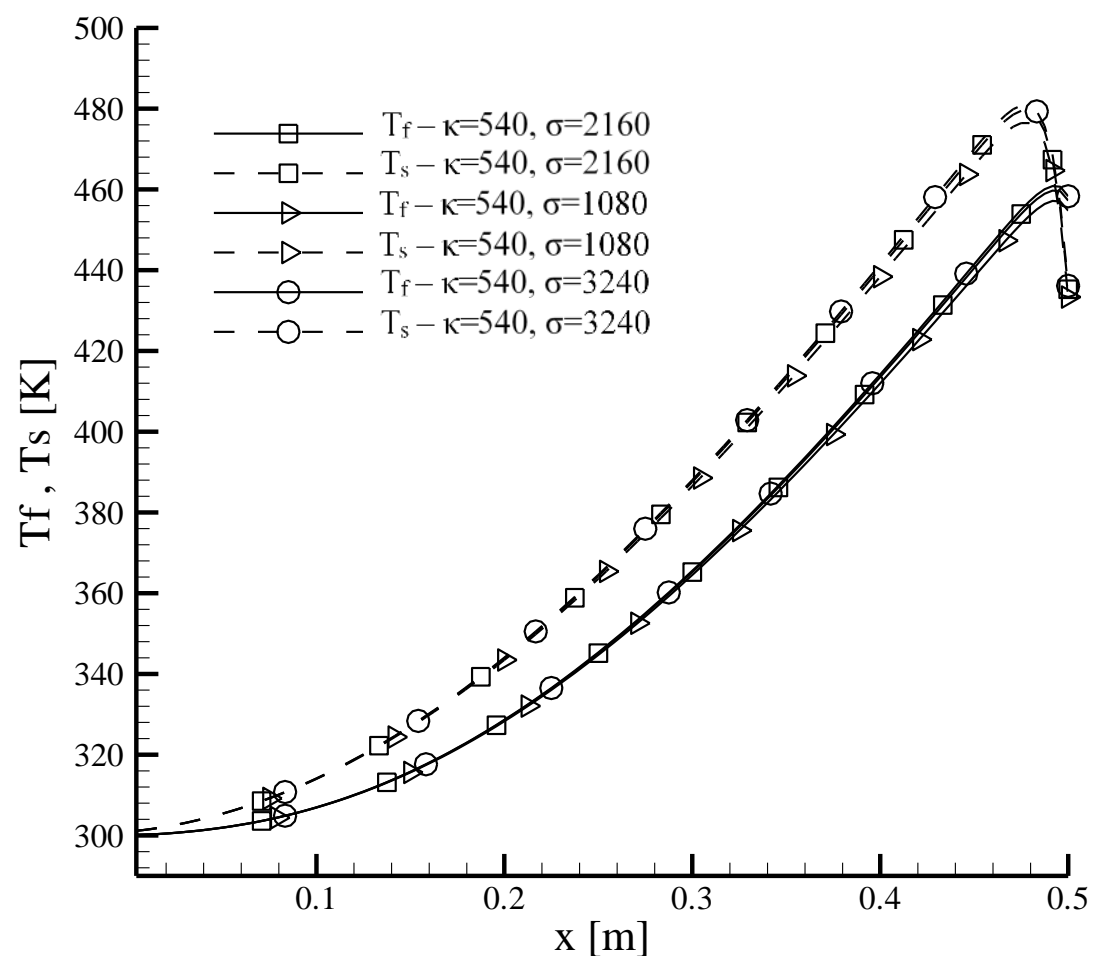

Figure 9. Temperature profiles of the fluid and solid phases in the center line of a porous channel obtained changing the scattering coefficient $\pm 50 \%$. It was utilized FVM with an angular discretization $\mathrm{N}_{\phi}=14$ and $\mathrm{N}_{\theta}=12$ considering isotropic scattering, $\mathrm{q}_{\mathrm{w}}=5000 \mathrm{~W} / \mathrm{m}^{2}$.

The sensitivity analyses was repeated considering another material, a 10 ppi oxidebonded silicon carbide ( $\mathrm{OB} \mathrm{SiC})$. The reason to repeat the analyses is to check the effect of perturbed properties considering a material with low values of absorption and scattering coefficients. The $\mathrm{OB} \mathrm{SiC}$ values adopted were $\kappa=150 \mathrm{~m}^{-1}$ and $\sigma=460 \mathrm{~m}^{-1}$, represent an average value over the wavelengths. The change in temperature results are presented in the Table 1 Case 2.

A third situation is considered in order to check the effect of the absorption coefficient on the temperature fields, the case considering zero albedo, or without scattering. The 
results are presented in Table 1 Case 3. It shows the highest variations in the temperature field. Compared to the other two cases studied, a difference of up to $-6.17 \%$ for a decrease of $50 \%$ in the $\kappa$ is observed.

Table 1. The maximum difference in the temperature field obtained with the perturbed optical properties

\begin{tabular}{|c|c|c|c|c|c|c|}
\hline & \multicolumn{2}{|c|}{$\begin{array}{c}\text { Case 1 } \\
\kappa=540 \mathrm{~m}^{-1}, \sigma=2160 \mathrm{~m}^{-1}\end{array}$} & \multicolumn{2}{|c|}{$\begin{array}{c}\text { Case 2 } \\
\kappa=150 \mathrm{~m}^{-1}, \sigma=460 \mathrm{~m}^{-1}\end{array}$} & \multicolumn{2}{|c|}{$\begin{array}{c}\text { Case 3 } \\
\kappa=540 \mathrm{~m}^{-1}, \sigma=0 \mathrm{~m}^{-1}\end{array}$} \\
\hline & $+\mathbf{5 0 \%}$ & $-50 \%$ & $+50 \%$ & $-50 \%$ & $+50 \%$ & $-50 \%$ \\
\hline$\kappa$ & $+1.24 \%$ & $-2.11 \%$ & $+1.38 \%$ & $-1.78 \%$ & $+2.73 \%$ & $-6.17 \%$ \\
\hline$\sigma$ & $+0.54 \%$ & $-1.22 \%$ & $+1.68 \%$ & $-3.32 \%$ & - & - \\
\hline
\end{tabular}

\subsection{The porous tube}

In this section, results are presented for simulations considering an axisymmetric cylindrical problem. Figure 10 represents a two-dimensional axisymmetric geometry corresponding to a porous tube. The fluid considered in all simulations is air. The length $\mathrm{L}$ is $50 \mathrm{~cm}$ and the diameter of the cross-section is $10 \mathrm{~cm}$.

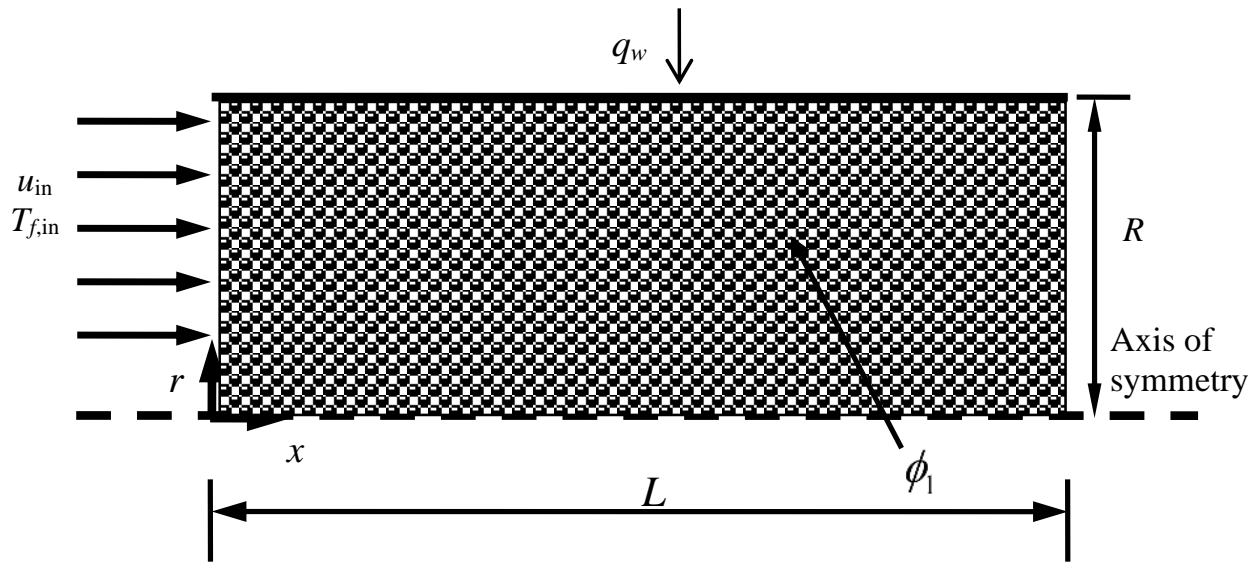

Figure 10. Geometry of a porous tube and coordinate system

In the literature, there are several publications presenting results for similar configurations using non-dimensional variables. These results are sometimes unsuitable for the comparisons with other studies because non-dimensionalisation itself tends to obscure the actual differences between the methods. In the work of Mahmoudi (2014), the nondimensional temperatures of the solid and fluid phase is defined as a non-dimensional 
parameter given by $\theta=\left(T-T_{i n}\right) /\left(q_{w} R / k_{f}\right)$. In the work of Yang and Vafai (2010) the non-dimensional temperatures of the solid and fluid phase are defined as $\theta=\left(T-T_{w}\right) /\left(q_{w} H / k_{s, e f f}\right)$. Figure 11 shows the comparison between the analytical results of Yang and Vafai (2010) with current numerical results presented using the nondimensional temperature $\theta$. When the solid and fluid phase temperatures are plotted utilizing these non-dimensional variables, the non-dimensional solid and fluid phase temperatures profiles obtained for a channel appear very close as that obtained for a tube. The same comparison was presented by Mahmoudi (2014) in order to validate his computational code.

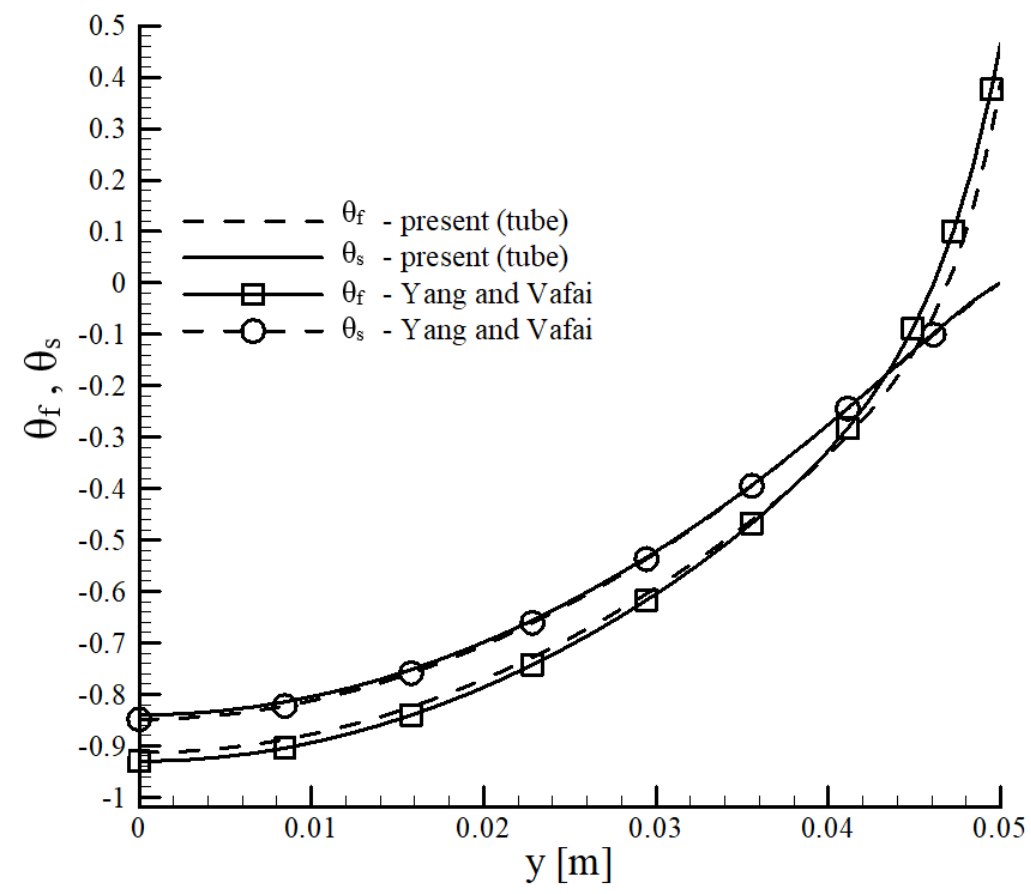

Figure 11. Numerical results for the fluid and solid temperatures along the tube radius and the exact fluid and solid temperatures obtained by Yang and Vafai (2010) for a channel in the fully developed region utilizing non-dimensional variables, $\mathrm{q}_{\mathrm{w}}=100$ $\mathrm{W} / \mathrm{m}^{2}$.

Figure 12 shows the actual solid phase and fluid phase temperatures for a tube and a channel from the present study presented without the non-dimensional parameters. It can be seen that the solutions of $\mathrm{T}_{\mathrm{s}}$ and $\mathrm{T}_{\mathrm{f}}$ for a tube and the solutions for a channel are very 
different as can be seen in the Figure 11. The problem with this non-dimensional temperature is that, intrinsically, a scale factor is being applied leading to a sense that the two solutions are close, in fact, they are not. While the maximum difference between the non-dimensional fluid temperatures is 0.017 and between the non-dimensional solid temperatures is 0.0087 , the maximum differences between the dimensional fluid temperatures is $20.37 \mathrm{~K}$ and between the dimensional solid temperatures is $20.17 \mathrm{~K}$. Therefore, the differences between the dimensional temperatures are more than one thousand times in the case of the fluid phase and more than two thousand times in the case of the solid phase. The different scales can lead to a wrong validation. The other problem with this non-dimensional variable is the indication that $\theta$ is independent of $\mathrm{q}_{\mathrm{w}}$. The radiation is a phenomenon related to the forth power of the temperature and this nondimensional variable do not reflect that. It would be more useful not to use nondimensional parameters to represent results and they should be presented as they are. This is evident from Figures 11 and 12.

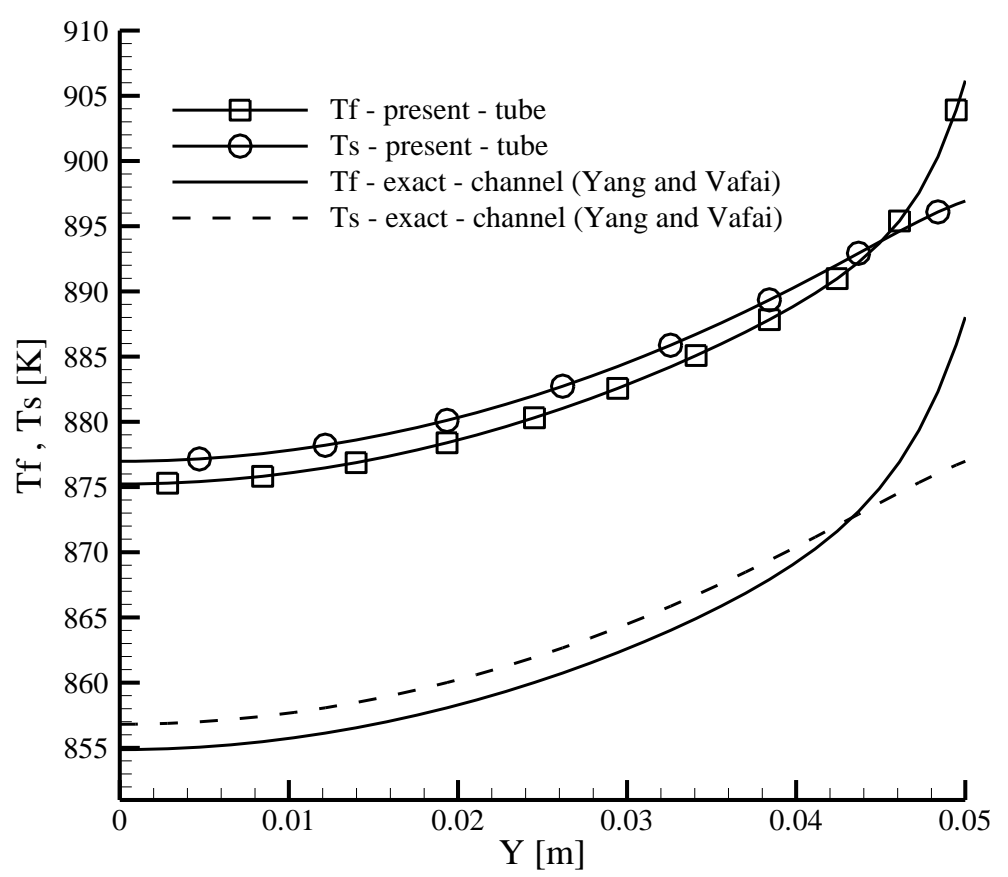

Figure 11: Comparison of the numerical results for the fluid and solid temperatures along the tube radius and the exact fluid and solid temperatures obtained by Yang and Vafai (2010) for a channel in the fully developed region, $\mathrm{q}_{\mathrm{w}}=100 \mathrm{~W} / \mathrm{m}^{2}$. 
The field temperatures of the fluid and solid phase are presented in Figure 12 with $\mathrm{q}_{\mathrm{w}}=5000 \mathrm{~W} / \mathrm{m}^{2}$. In the calculations, the spatial domain was divided into $222 \times 34$ control volumes with the mesh refined close to the wall and in the inlet and exit regions. The R.T.E. was solved through the discrete ordinate method with an S12 quadrature. The step scheme was used in all simulations.

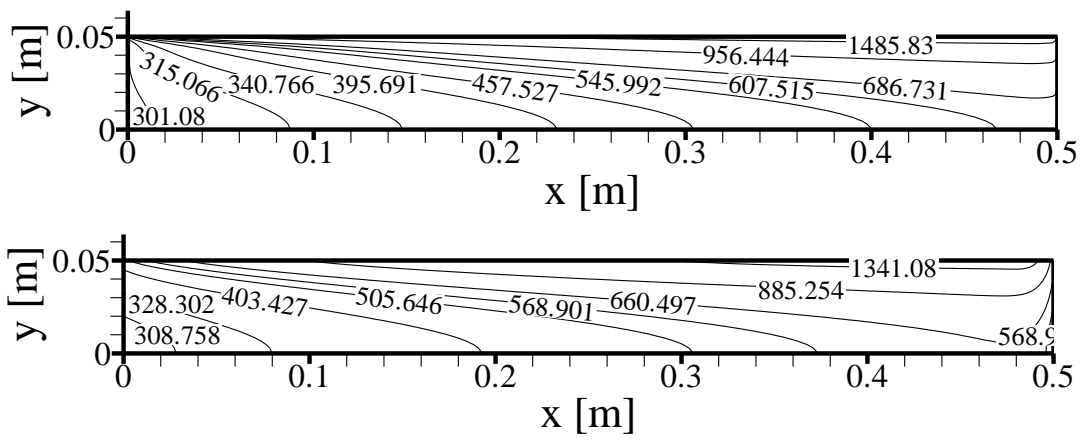

Figure 12. Fields of predicted temperature $(\mathrm{K})$ of the fluid (a) and solid (b) phase in a tube obtained applying DOM with $\mathrm{S} 12$ quadrature, $\mathrm{q}_{\mathrm{w}}=5000$ $\mathrm{W} / \mathrm{m}^{2}$.

The divergent of the radiative heat flux, which appears as a source term accounting the radiative heat transport in the solid energy equation, depends on the incidence of the radiation. In order to ensure correct values to the $\vec{\nabla} \cdot \vec{q}_{r}$, it was necessary to develop a mesh analysis observing the gradients on the fields of the incidence of the radiation and of the divergent of the radiative heat flux. Both variables present high gradients that can affect the convergence and the results. The field of the predicted incidence radiation is presented in Figure 13 (a) and the field of the divergent of the radiative heat flux is presented in Figure $13(b)$. 

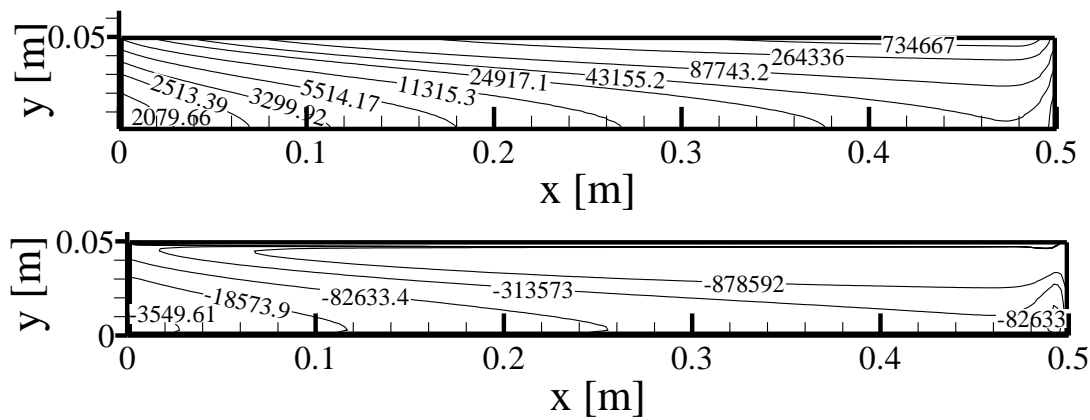

Figure 13. Field of predicted incidence radiation (a) and field of predicted divergence of the radiative heat flux (b) in a tube obtained through the method of DOM S12, $\mathrm{q}_{\mathrm{w}}=5000 \mathrm{~W} / \mathrm{m}^{2}$.

The results obtained with DOM, Rosseland model and non-radiative model are presented in Figure 14. The fields of solid and fluid phase temperatures obtained with Rosseland are higher than that obtained with DOM except in the region close to the wall. This is because in the Rosseland model the heat diffusion is increased providing an increase in the heat transport from the wall to the center of the tube. It is the reason that the temperatures are so high in the center line when obtained through the Rosseland model. The differences between the temperatures calculated with DOM and with Rosseland are up to $75.98 \%$ in the region far from the wall and up to $-22.30 \%$ close to the wall.

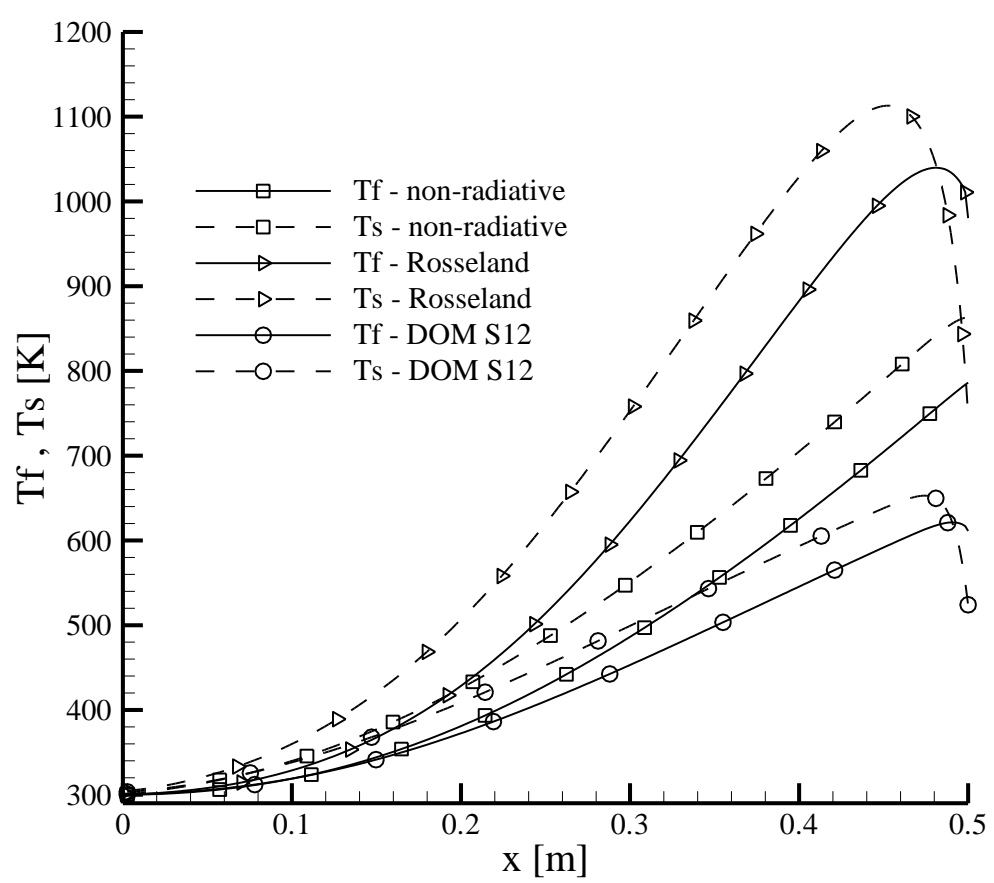

Figure 14: Comparison of the numerical results for the fluid and solid temperatures obtained with DOM, Rosseland model and nonradiative model in the center line of the tube, $\mathrm{q}_{\mathrm{w}}=5000 \mathrm{~W} / \mathrm{m}^{2}$. 
The comparison between the temperatures fields obtained with DOM and with the non-radiative model shows differences of up to $+155.63 \%$ and differences of up to $36.22 \%$. The high temperatures obtained with DOM, when compared with the nonradiative model, were found in the region close to the wall near the inlet. Although there is a loss of radiation in the inlet region, there is a gain by radiation coming from downstream. This gain makes with the temperatures obtained with DOM are higher than that obtained with the non-radiative model in the region close to the wall near the inlet.

The Figure 15 presents the obtained values for the Nusselt number for different extinction coefficients $(\beta)$ considering a tube filled with a porous material with a porosity of 0.9. The R.T.E. was solved with an S12 quadrature. For a clean medium (without a porous medium) the Nusselt number obtained was 4.41 , close to the exact value 4.36 (Incropera et al. 2007). The Nusselt obtained for the porous tube without considering radiation was 20.87 .

The analysis of the field of the incidence radiation can help to understand the results presented in Figure 15. The results show that the field of the incidence radiation increase with $\beta$. The higher the values of $\beta$ more heterogeneous is the field of the incident radiation, with the higher values close to the hot wall and the lower values far from it. The field of the incidence radiation is homogeneous for very low $\beta$, approaching the optically thin limit. Increasing $\beta$, the radiation travels shorter distances before being completely attenuated and the incidence radiation in a point presents a strong dependence on the local temperatures. This is the reason that as higher the value of the $\beta$ more the shape of the isolines, relative to the field of the incident radiation, looks like the shape of the isolines of the temperatures. For the extinction coefficient higher than 200, the radiation becomes more dependent on the local temperatures and the Nusselt increases with the increase of $\beta$. For values of $\beta$ lower than 200 , the Nusselt decrease with the increase of $\beta$. This happens 
because, for very low $\beta$, lower than 200 , the radiation travels long distances, spreading energy far from the hot wall. For very low $\beta$, the temperature profile in the cross-section of the tube, in a completely developed flow, presents lower temperatures. For high values of $\beta$, the temperature profile increases approaching the temperature profile obtained for the case not considering radiation. For $\beta$ in the range between 0 and 200 the Nusselt decrease with the increase of $\beta$. For $\beta$ higher than 200 , the Nusselt increase with the increase of $\beta$.

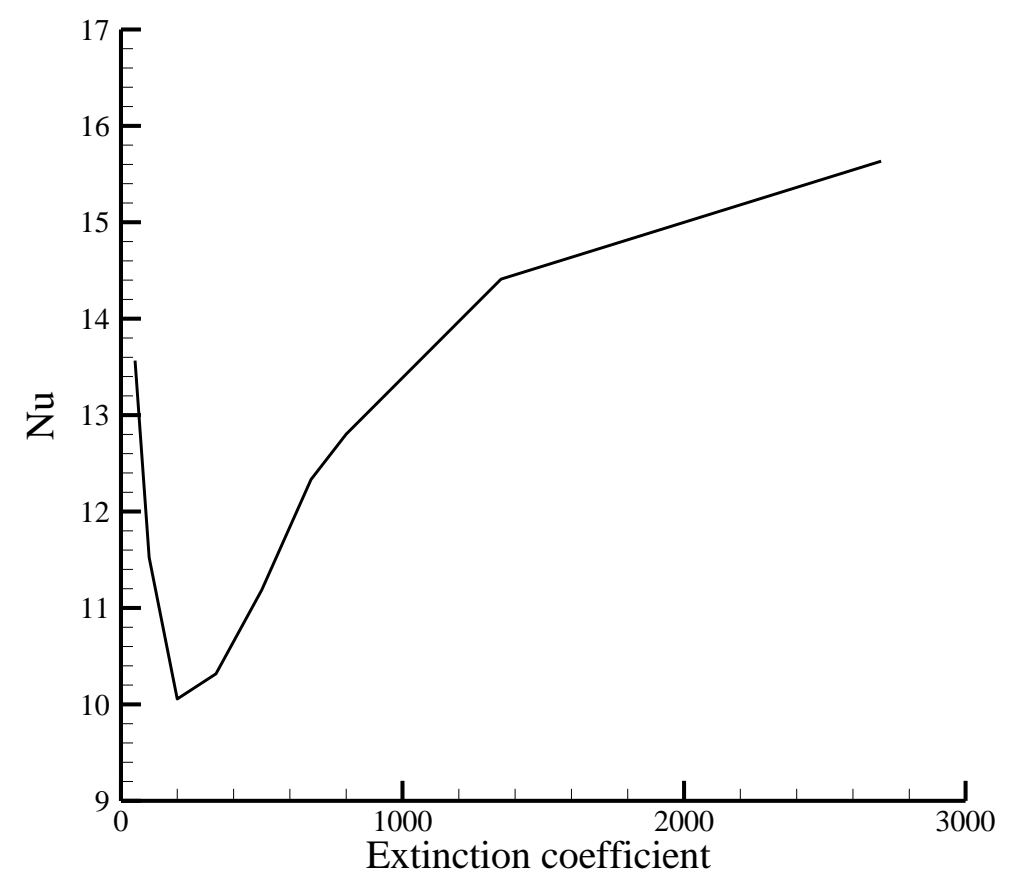

Figure 15: $\mathrm{Nu}$ obtained for different extinction coefficients for a tube in the fully developed region.

\section{Conclusion}

This research has presented the effect of the radiation heat transfer in porous media applications involving tubes and channels and showed that the results are sometimes unsuitable for the comparisons with other studies because non-dimensionalization itself tends to obscure the actual differences between the methods. A sensitivity analysis was performed to determine the importance of the radiation and optical properties of the porous tubes and porous channels. The modelling of radiation considered the solid and fluid phases as a single continuum homogeneous medium. Two different methods were applied 
to solve the radiative transfer equation, the discrete ordinate method (DOM) and the finite volume method (FVM). The results of the two methods are very close when compared with S12 with an angular discretization of $14 \times 12$, maximum difference of $0.26 \%$. Some significant differences can only be seen when the methods are applied with a low number of angular directions.

The results obtained with DOM and with FVM were compared with the results obtained considering the diffusion approximation (Rosseland Model). In the cases with Rosseland approximation, the effective conductivity is increased with the inclusion of the Rosseland diffusion term. The increased effective conductivity provides high heat transfer from the wall to the center of the channel or tube, leading to a drop in the temperatures close to the wall. The temperatures close to the wall are very low in the cases solved through Rosseland model when compared with the cases solved with FVM and DOM. In the middle of the tube or channel takes place the opposite, the temperatures obtained with Rosseland are pretty much higher than that obtained through DOM or FVM. The maximum difference between the temperature fields obtained through the diffusion approximation and the temperature fields obtained solving the R.T.E., was $75.98 \%$, what proves that the Rosseland Model provides very different results. The diffusion approximation is not valid near the boundaries where the model loses accuracy. In pipes and channels with high heat flux on the walls the model fails.

The absorption and the scattering coefficient were perturbed $\pm 50 \%$ causing small changes in the temperature fields. The maximum difference in the temperature field, in the cases considering isotropic scattering, was 3.32\%. The differences were higher in the cases considering a medium that absorbs and emits but does not scatter, differences of up to $6.17 \%$. 
A numerical investigation on the effect of the extinction coefficient on the Nusselt number for a porous tube was presented in this work. The results showed that for $\beta$ in the range between 0 and 200, the Nusselt number decreased with the increase of $\beta$. For $\beta$ higher than 200, the Nusselt increased with the increase of $\beta$. The highest values of the Nusselt number for a porous tube were obtained considering very low and very high values of $\beta$, in the limits tending to the non-radiative model. The maximum Nusselt number was obtained with a non-radiative model.

\section{Acknowledgments}

The authors are thankful to CNPQ, Brazil, for their financial support during the preparation of this work and to the Loughborough University for providing the facilities, equipment, all the support necessary.

Table 2. Operating Conditions

\begin{tabular}{cc}
\hline Quantity & Value \\
\hline $\mathrm{c}_{\mathrm{F}}$ & 0.55 \\
$\mathrm{~d}_{\mathrm{p}}(\mathrm{m})$ & 0.008 \\
$\mathrm{u}_{\text {in }}(\mathrm{m} / \mathrm{s})$ & 0.17 \\
$\mathrm{~T}_{\infty}(\mathrm{K})$ & 300 \\
$\mathrm{~T}_{\text {in }}(\mathrm{K})$ & 300 \\
Length of the tube $-\mathrm{L}(\mathrm{cm})$ & 50 \\
Diameter of the Tube - D $(\mathrm{cm})$ & 5 \\
Length of the channel $-\mathrm{L}(\mathrm{cm})$ & 50 \\
Height of the channel $(\mathrm{cm})$ & 10 \\
$\mathrm{~K}\left(\mathrm{~m}^{2}\right)$ & $1.477 \times 10^{-8}$ \\
$\phi$ & 0.9 \\
$a_{v 1}$ & $1060 \mathrm{~m}^{-1}$ \\
$\mathrm{k}_{\mathrm{s}} / \mathrm{k}_{\mathrm{f}}$ & 80 \\
\hline
\end{tabular}

\section{References}

Chaffin, C., Koenig, M., Koeroghlian, M., Matthews, R. D., Hall, M.J., Nichols, S.P., Lim, I.-G.: Experimental Investigation of Premixed Combustion within Highly Porous Media." ASME/JSME Thermal Eng. Conf. 4:219-24, (1991). 
Chai, J. C., Lee, H.S., Patankar, S.V.: Finite Volume Method for Radiation Heat Transfer. Journal of Thermophysics and Heat Transfer 8 (3):419-25 (1994).

Chandrasekhar, S.: Radiative Transfer. New York: Dover (1960)

Chui, E. H., Raithby, G.D., Hughes, P.M.J.: Prediction of Radiative Transfer in Cylindrical Enclosures with the Finite Volume Method. Journal of Thermophysics and Heat Transfer 6 (4):605-11 (1992). https://doi.org/10.2514/3.11540.

Dullien, F.A.L.: Porous Media: Fluid Transport and Pore Structure. 2nd ed. Academic Press (1991)

Fiveland, W. A.: Discrete-Ordinates Solutions of the Radiative Transport Equation for Rectangular Enclosures. Journal of Heat Transfer 106 (4). American Society of Mechanical Engineers:699 (1984) https://doi.org/10.1115/1.3246741.

Frederking, T. H. K., Hepler, W. A., Yuan, S. W. K., Feng, W. F.: Determination of the Darcy Permeability of Porous Media Including Sintered Metal Plugs. In Advances in Cryogenic Engineering, 505-15. Boston, MA: Springer US (1986). https://doi.org/10.1007/978-1-4613-2213-9_58.

Fu, X., Viskanta, R., Gore, J. P.: Measurement and Correlation of Volumetric Heat Transfer Coefficients of Cellular Ceramics. Experimental Thermal and Fluid Science 17:285-93 (1998)

Happel, J., Brenner, H.: Low Reynolds Number Hydrodynamics. Vol. 1. Mechanics of Fluids and Transport Processes. Dordrecht: Springer Netherlands (1981). https://doi.org/10.1007/978-94-009-8352-6.

Howell, J., Siegel, R., Mengüç, M. P.: Thermal Radiation Heat Transfer. CRC Press (2011)

Hwang, G. J., Chao, C. H.: Heat Transfer Measurement and Analysis for Sintered Porous Channels. Journal of Heat Transfer 116 (2). American Society of Mechanical Engineers:456 (1994) https://doi.org/10.1115/1.2911418.

Imani, G. R., Maerefat, M., Hooman, K.: Estimation of Heat Flux Bifurcation at the Heated Boundary of a Porous Medium Using a Pore-Scale Numerical Simulation. International Journal of Thermal Sciences 54. Elsevier Masson:109-18 (2012) https://doi.org/10.1016/J.IJTHERMALSCI.2011.11.006.

Incropera, F. P, DeWitt, D.P., Bergman, T.L., Lavine, A. S.: Fundamentals of Heat and Mass Transfer. Wiley. Vol. $6^{\text {th }}$ (2007)

Jiang, Pei-Xue, Meng Li, Tian-Jian Lu, Lei Yu, and Ze-Pei Ren. 2004. "Experimental Research on Convection Heat Transfer in Sintered Porous Plate Channels." International Journal of Heat and Mass Transfer 47 (10-11). Pergamon:2085-96. https://doi.org/10.1016/J.IJHEATMASSTRANSFER.2003.12.004.

Kaviany, M.: Principles of Heat Transfer in Porous Media. Mechanical Engineering Series. New York, NY: Springer New York (1995) https://doi.org/10.1007/978-14612-4254-3.

Kuwahara, F., Kameyama, Y. Yamashita,S., Nakayama, A.: Numerical Modeling of Turbulent Flow in Porous Media Using a Spatially Periodic Array. Journal of Porous Media 1 (1). Begel House Inc.:47-55 (1998) https://doi.org/10.1615/JPorMedia.v1.i1.40.

Kuwahara, F., Shirota,M. ,Nakayama, A.: A Numerical Study of Interfacial Convective Heat Transfer Coefficient in Two-Energy Equation Model for Convection in Porous Media. International Journal of Heat and Mass Transfer 44 (6):1153-59 (2001) https://doi.org/10.1016/S0017-9310(00)00166-6.

Mahmoudi, Y.: Effect of Thermal Radiation on Temperature Differential in a Porous Medium under Local Thermal Non-Equilibrium Condition. International Journal of Heat and Mass Transfer 76:105-21 (2014) 
https://doi.org/10.1016/j.ijheatmasstransfer.2014.04.024.

Malico, I., Zhou, X. Y., Pereira, J. C. F.: Two-Dimensional Numerical Study of Combustion and Pollutants Formation in Porous Burners. Combustion Science and Technology 152 (1):57-79 (2000) https://doi.org/10.1080/00102200008952127.

Miguel, A. F., Serrenho, A.: On the Experimental Evaluation of Permeability in Porous Media Using a Gas Flow Method. Journal of Physics D: Applied Physics 40 (21). IOP Publishing:6824-28 (2007) https://doi.org/10.1088/0022-3727/40/21/050.

Modest, M. Radiative Heat Transfer. 3rd Ed. Academic Press (2013)

Moro Filho, R. C.: Modeling of Premixed Combustion on Inert Porous Media.” In Proceedings of the Ninth International Conference on Engineering Computational Technology, CivilComp Press (2014) https://doi.org/10.4203/ccp.105.80.

Moro Filho, R. C., Pimenta, A.P.: A numerical study of combustion in porous burner with a two-energy equation model. In 20th International Congress of Mechanical Engineering. Gramado: ABMC (2009)

Moro Filho, R. C., Pimenta, A.P.: A Two-Dimensional Numerical Simulation of Combustion and Heat Transfer in Radiant Porous Burners. Combustion Science and Technology 183 (4):370-89 (2011) https://doi.org/10.1080/00102202.2010.520278.

Nield, D. A., Bejan, A.: Convection in Porous Media. New York, NY: Springer New York (2013) https://doi.org/10.1007/978-1-4614-5541-7.

Niya,R. S. M., Selvadurai, A. P. S.: The Estimation of Permeability of a Porous Medium with a Generalized Pore Structure by Geometry Identification. Physics of Fluids 29 (3). AIP Publishing LLC:37101 (2017) https://doi.org/10.1063/1.4977444.

Ouyang, X.L., Vafai, K. Jiang, P.X.: Analysis of Thermally Developing Flow in Porous Media under Local Thermal Non-Equilibrium Conditions. International Journal of Heat and Mass Transfer 67 (December). Pergamon:768-75 (2013)

Ozgumus, T., Mobedi, M. Ozkol, U.: Determination of Kozeny Constant Based on Porosity and Pore to Throat Size Ratio in Porous Medium with Rectangular Rods. Engineering Applications of Computational Fluid Mechanics 8 (2). Taylor \& Francis:308-18 (2014) https://doi.org/10.1080/19942060.2014.11015516.

Patankar, S V.: Numerical heat transfer and fluid flow. New York: Hemisphere (1980)

Petrasch, R., Meier,J.F., Friess, H., Steinfeld, A.: Tomography Based Determination of Permeability, Dupuit-Forchheimer Coefficient, and Interfacial Heat Transfer Coefficient in Reticulate Porous Ceramics (2007) https://doi.org/10.1016/j.ijheatfluidflow.2007.09.001.

Raithby, G. D., Chui, E. H.: A Finite-Volume Method for Predicting a Radiant Heat Transfer in Enclosures With Participating Media. Journal of Heat Transfer 112 (2). American Society of Mechanical Engineers:415 (1990) https://doi.org/10.1115/1.2910394.

Schuster, A.: Radiation Through a Foggy Atmosphere. The Astrophysical Journal 21 (January):1 (1905) https://doi.org/10.1086/141186.

Schwarzschild, K.: On the Equilibrium of the Sun's Atmosphere. Nachrichten von Der K\{ö\}niglichen Gesellschaft Der Wissenschaften Zu G\{ö\}ttingen. Math.-Phys. Klasse, 195, p. $41-53$ 195:41-53 (1906)

Shah, N. New Method of Computation of Radiation Heat Transfer in Combustion Chambers. Imperial College of Science and Technology, University of London (1979)

Stone, H. L.: Iterative Solution of Implicit Approximations of Multidimensional Partial Differential Equations. SIAM Journal on Numerical Analysis 5 (3):530-58 (1968) https://doi.org/10.1137/0705044.

Truelove, J. S.: Discrete-Ordinate Solutions of the Radiation Transport Equation. Journal of Heat Transfer 109 (4):1048-51 (1987) https://doi.org/10.1115/1.3248182. 
Versteeg, H.K. and Malalasekera, W.: An Introduction to Computational Fluid Dynamics: The Finite Volume Method." Pearson Education (2007)

Wakao, N., Kagei, S.: Heat and Mass Transfer in Packed Beds. Gordon and Breach Science Publishers (1982)

Wang, P., K. Vafai, Liu, D. Y.: Analysis of Radiative Effect under Local Thermal NonEquilibrium Conditions in Porous Media-Application to a Solar Air Receiver. Numerical Heat Transfer, Part A: Applications 65 (10). Taylor \& Francis Group :931-48 (2014) https://doi.org/10.1080/10407782.2013.850917.

Ward, J C. Turbulent Flow in Porous Media.” Proc. Amer. Soc. Civil Eng. No. HY5 90:1$12(1964)$

Yang, K., Vafai, K.: Analysis of Temperature Gradient Bifurcation in Porous Media - An Exact Solution. International Journal of Heat and Mass Transfer 53 (19):4316-25 (2010) https://doi.org/10.1016/j.ijheatmasstransfer.2010.05.060.

Yang, K., Vafai, K.: Analysis of Heat Flux Bifurcation inside Porous Media Incorporating Inertial and Dispersion Effects - An Exact Solution. International Journal of Heat and Mass Transfer 54:5286-97 (2011) 